\title{
Cytomorphological Features of Hyperchromatic Crowded Groups in Liquid-Based Cervicovaginal Cytology: A Single Institutional Experience
}

\author{
Youngeun Lee, Cheol Lee, In Ae Park, Hyoung Jin An, Haeryoung Kim \\ Department of Pathology, Seoul National University Hospital, Seoul National University College of Medicine, Seoul, Korea
}

\begin{abstract}
Background: Hyperchromatic crowed groups (HCGs) are defined as three-dimensional aggregates of crowded cells with hyperchromatic nuclei, and are frequently encountered in cervicovaginal liquid-based cytology (LBC). Here, we aimed to examine the prevalence of HCGs in cervicovaginal LBC and the cytomorphological characteristics of various epithelial cell clusters presenting as HCGs. Methods: We first examined the prevalence of HCGs in a "routine cohort" of LBC cytology $(n=331)$, consisting of all cervicovaginal LBCs accessioned over 3 days from outpatient clinics $(n=179)$ and the screening population $(n=152)$. Then we examined a second "high-grade epithelial cell abnormalities (H-ECA) cohort" ( $n=69$ ) of LBCs diagnosed as high-grade squamous intraepithelial lesion (HSIL), squamous cell carcinoma (SCC), or adenocarcinoma during 1 year. Results: HCGs was observed in $34.4 \%$ of the routine cohort and were significantly more frequent in the epithelial cell abnormality category compared to the non-neoplastic category $(p=.003)$. The majority of HCGs represented atrophy (70\%). Of the 69 histologically confirmed H-ECA cases, all contained HCGs. The majority of cases were HSIL $(62 \%)$, followed by SCC (16\%). Individually scattered neoplastic cells outside the HCGs were significantly more frequent in SCCs compared to glandular neoplasia $(p=.002)$. Despite the obscuring thick nature of the HCGs, examining the edges and the different focal planes of the HCGs and the background were helpful in defining the nature of the HCGs. Conclusions: HCGs were frequently observed in cervicovaginal LBC and were mostly non-neoplastic; however, neoplastic HCGs were mostly high-grade lesions. Being aware of the cytomorphological features of different HCGs is important in order to avoid potential false-negative cytology interpretation.
\end{abstract}

Key Words: Hyperchromatic crowded groups; Cervical neoplasms; Liquid-based cytology

Received: July 11, 2019 Revised: August 13, 2019 Accepted: August 14, 2019

Corresponding Author: Haeryoung Kim, MD, PhD, Department of Pathology, Seoul National University Hospital, Seoul National University College of Medicine, Basic Science

Building 101, 103 Daehak-ro, Jongno-gu, Seoul 03080, Korea

Tel: +82-2-740-8322, Fax: +82-2-765-5600, E-mail: haeryoung.kim@snu.ac.kr

Hyperchromatic crowded groups (HCGs, also known as "microbiopsies") are defined as three-dimensional aggregates of crowded cells with hyperchromatic nuclei. ${ }^{1}$ They are commonly found in liquid-based cytology (LBC) samples-in almost $80 \%$ according to one study ${ }^{2}$ —and are easily detected at low-power magnification.

Most of the time, HCGs represent normal or reactive conditions, such as endometrial cells, reactive endocervical cells, basal cells in severe atrophy, squamous metaplasia or tubal metaplasia, or even non-epithelial components such as neutrophil clusters, lymphoid follicles or bacterial colonies. ${ }^{3}$ However, they may also occasionally represent the other extreme of the benign-malignant spectrum, including high-grade squamous intraepithelial lesions (HSIL), squamous cell carcinomas, or glandular neoplasia. Thus, it is important to bear in mind that HCGs require careful examination before being passed off as normal/benign. In fact, HCGs have been reported to be the most common reason for false-negative reports in cervicovaginal LBC. ${ }^{4}$

In this study, we aimed to evaluate the prevalence of HCGs in cervicovaginal LBC and examine the cytomorphological characteristics of various epithelial cell clusters presenting as HCGs.

\section{MATERIALS AND METHODS}

\section{Subjects}

In order to examine the prevalence of HCG in everyday practice, we first reviewed all cervicovaginal LBC slides accessioned at Seoul National University Hospital during 3 days in January 2012 ("routine cohort"), including specimens from the outpatient clinics and from the screening population (health promo- 
tion center). All LBC slides were prepared by the BD SurePath system (BD Diagnostics, Burlington, NC, USA).

We collected a second cohort ("high-grade epithelial cell abnormalities [H-ECA] cohort") of all cervicovaginal LBC cases diagnosed as HSIL, squamous cell carcinoma, adenocarcinoma, or atypical glandular cell (AGC) from January 2012 to December 2012 at Seoul National University Hospital, in order to examine in more detail the cytomorphological characteristics of neoplastic HCGs. Among a total of 1,130 retrieved cases, we included only cases that were confirmed by histological examination (concurrent or subsequent biopsies or operative specimens), and we also excluded all conventional smears. The remaining 69 cases were subjected to detailed cytomorphological review and the patient age information was obtained from the pathology database.

\section{Cytological review}

All the cervicovaginal smears were reviewed by two pathologists (H.K. and Y.L.). The cytological details examined included the cytological diagnosis, presence of endocervical/transformation zone (EC/TZ) components, presence and the nature (neoplastic versus non-neoplastic) of HCGs, and the presence of scattered single neoplastic cells in the background. We defined HCGs as dense cellular aggregates of at least 10 cells and excluded HCGs consisting of neutrophil clusters and bacterial colonies.

\section{Statistical analyses}

Statistical analyses were performed using SPSS statistics ver. 25.0 (IBM Corp., Armonk, NY, USA). The student t-test, Pearson chi-square test, and Fisher exact test were used. A p-value of less than .05 was considered statistically significant.

\section{Ethics statement}

This study was approved by the Institutional Review Board of Seoul National University Hospital (\#H-1905-090-1034) and informed consent was waived due to the retrospective nature of the study.

\section{RESULTS}

\section{Prevalence of HCGs in routine cervicovaginal LBC}

A total of 331 cases were collected in the routine cohort, of which 152 cases (45.9\%) were from the screening population and the remaining 179 cases $(54.1 \%)$ were from outpatient clinics. The cases consisted of eight cases (2.4\%) and 323 cases (97.6\%) in the ECA and negative for intraepithelial lesion or malignancy (NILM) categories, respectively (Fig. 1). The ECA cases of the routine cohort consisted of one squamous cell carcinoma, one adenocarcinoma, one AGC, one low-grade squamous intraepithelial lesion (LSIL), and four atypical squamous cells of uncertain significance (ASC-US). The AGC case was subsequently confirmed as endocervical adenocarcinoma by histology. In the routine cohort, HCGs were observed in 114 cases (34.4\%). The frequency of HCGs was significantly higher in the ECA category $(7 / 8,87.5 \%)$ compared to the NILM category $(107 / 323$, $33.1 \%$; $\mathrm{p}=.003)$.

The majority of HCGs in the NILM group comprised atrophic parabasal cell clusters $(\mathrm{n}=75,70.1 \%)$, and the remainder were endocervical or metaplastic cells $(\mathrm{n}=25,23.4 \%)$ and endometrial cells $(n=7,6.5 \%)$. The HCGs in atrophy were characterized by flat monolayer sheets of parabasal cells with nuclear overlapping in individual focal planes. The constituent parabasal cells had dark nuclei and scant cytoplasm, but the nuclei showed regular nuclear contours and were frequently even, smudgy and degenerated (Fig. 2A). Maturation of parabasal cells at the edges of HCGs and the streaming pattern with preserved polarity were helpful clues in classifying the HCGs as atrophic.

Endocervical cells frequently presented as a large HCG fragments, and focusing up and down revealed the regular honeycomb arrangement with distinct cytoplasmic borders (Fig. 2B). Reactive endocervical cells often demonstrated prominent nucleoli, mild nuclear size variation and even occasional mitotic figures (Fig. 2C). However, the presence of a streaming pattern, smooth and round nuclear outlines and mild nuclear hyperchromasia were indicative of reactive cellular changes. Metaplastic HCGs showed dense cytoplasm and spindled cytoplasmic projections.

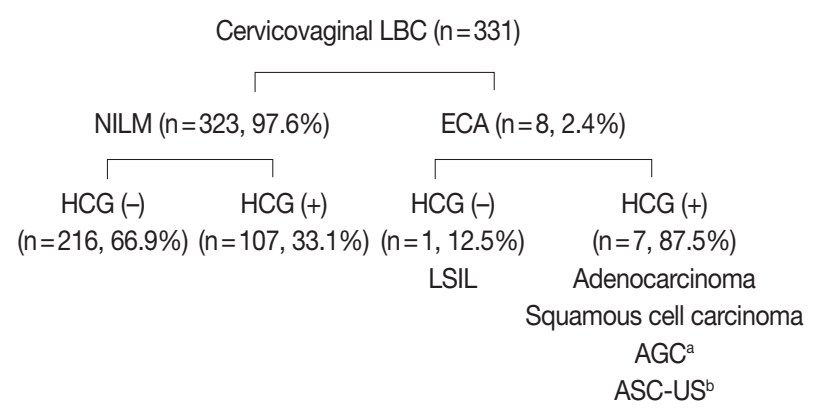

Fig. 1. Summary of the routine cervicovaginal liquid-based cytology (LBC) cases. Hyperchromatic crowed groups (HCGs) were significantly more frequent in the epithelial cell abnormality (ECA) category compared to the negative for intraepithelial lesion or malignancy (NILM) category, and neoplastic HCGs were high-grade lesions. LSIL, low-grade squamous intraepithelial lesion; AGC, atypical glandular cell; ASC-US, atypical squamous cells of uncertain significance. 'a Subsequently diagnosed as adenocarcinoma; ' ${ }^{\text {Only non- }}$ neoplastic HCGs were observed. 

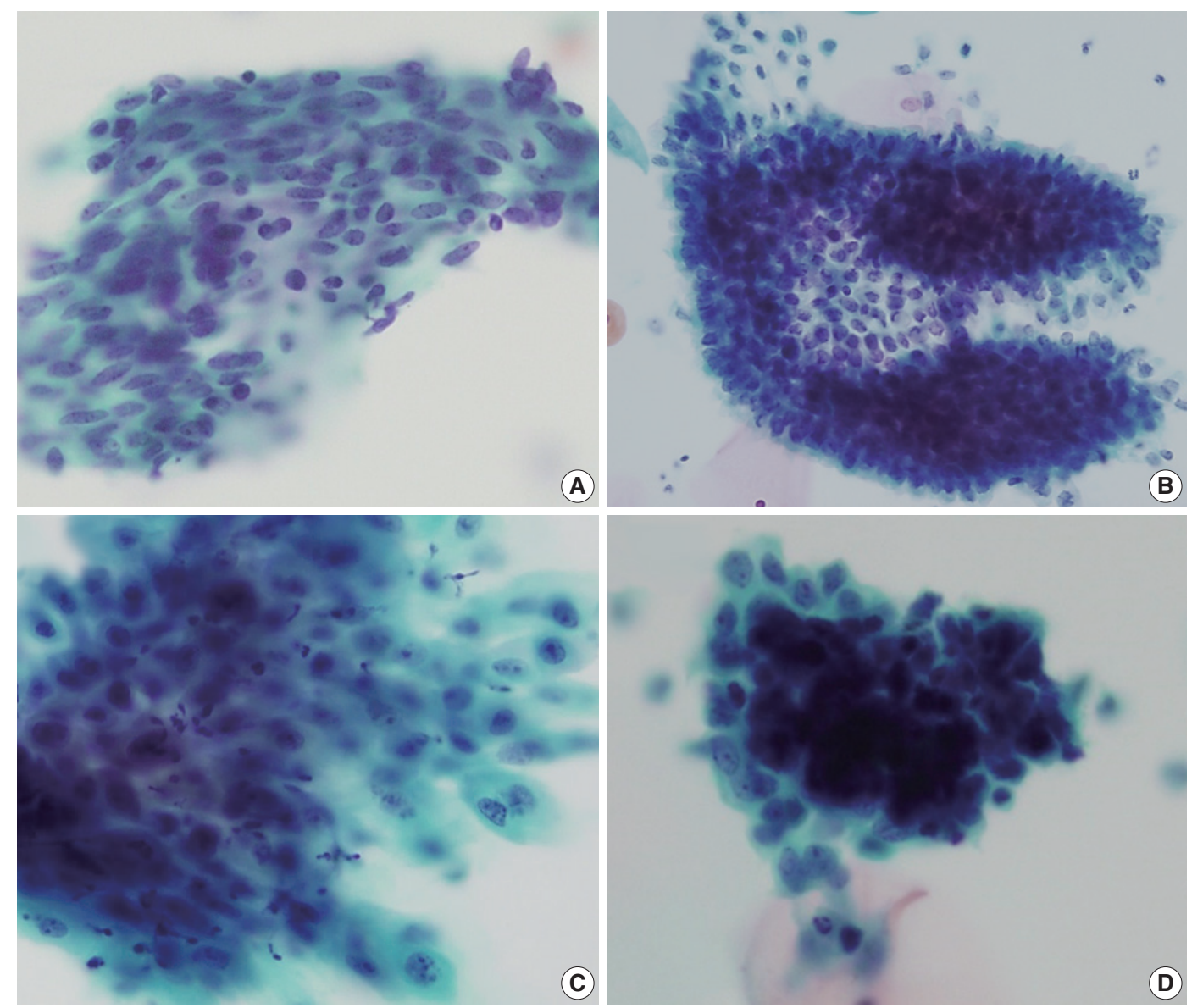

Fig. 2. Cytological features of non-neoplastic hyperchromatic crowed group (HCG). (A) Atrophic HCGs consisted of bland-looking parabasal cells with streaming patterns. (B) Regular honeycomb arrangement of endocervical cells. (C) Reactive endocervical cells demonstrating mild nuclear size variation and nucleoli but smooth nuclear membranes. (D) Biphasic pattern observed in endometrial cell clusters.

Endometrial cells appeared as very dense cellular clusters, and although the centers of HCGs were too dark to appreciate the cytomorphology even by examining different focal planes, the biphasic gland-stroma pattern was a helpful feature for identifying the endometrial cells (Fig. 2D). The periphery of the HCGs were lined by glandular cells with high nuclear:cytoplasmic ratio and small, hyperchromatic nuclei.

In the ECA group, all HCGs in the squamous cell carcinoma and glandular lesions were neoplastic HCGs. However, all cases diagnosed as ASC-US ( $n=4)$ demonstrated only non-neoplastic HCGs, and there were no HCGs in the LSIL case. All neoplastic HCGs were seen in the outpatient clinic setting, and were not seen in the general screening population.

As expected, the mean age of subjects with atrophic HCGs ( $62.8 \pm 7.9$ years) were significantly higher compared to those with endometrial HCGs $(39.5 \pm 4.8$ years) in the NILM group $(\mathrm{p}<.001)$. Interestingly, EC/TZ components were present in all 114 HCG-positive cases, while 34 of 217 HCG-negative cases
(15.7\%) did not contain EC/TZ components ( $\mathrm{p}<.001)$. On comparing the screening and outpatient populations, no statistically significant differences were seen in the age distribution or the frequency of HCGs.

\section{Prevalence and characteristics of neoplastic HCGs}

All 69 cases of the H-ECA cohort contained HCGs. The HECA cohort consisted of 63 uterine lesions (91.3\%) (HSIL [ $\mathrm{n}=$ 43], squamous cell carcinoma $[n=11]$, endometrial cancer $[n=$ $7]$, endocervical cancer $[n=2]$ ) and six extrauterine $(8.7 \%)$ (ovarian $[n=4]$, colorectal $[n=2]$ ) lesions (Fig. 3). The endometrial lesions comprised four endometrioid carcinomas, one clear cell carcinoma, one serous carcinoma, and one carcinosarcoma. All extrauterine lesions were adenocarcinomas.

All HCGs (100\%) in the glandular neoplasia were neoplastic HCGs. In contrast, in the squamous lesions, neoplastic HCGs were observed in 45 cases (83.3\%). Neoplastic HCGs were seen in all of the squamous cell carcinomas, while they were seen in 34 
of HSILs (79.1\%). The remaining nine (20.9\%) HSILs showed individually scattered HSIL cells without neoplastic HCGs.

Neoplastic squamous HCGs (HSIL and squamous cell carci-

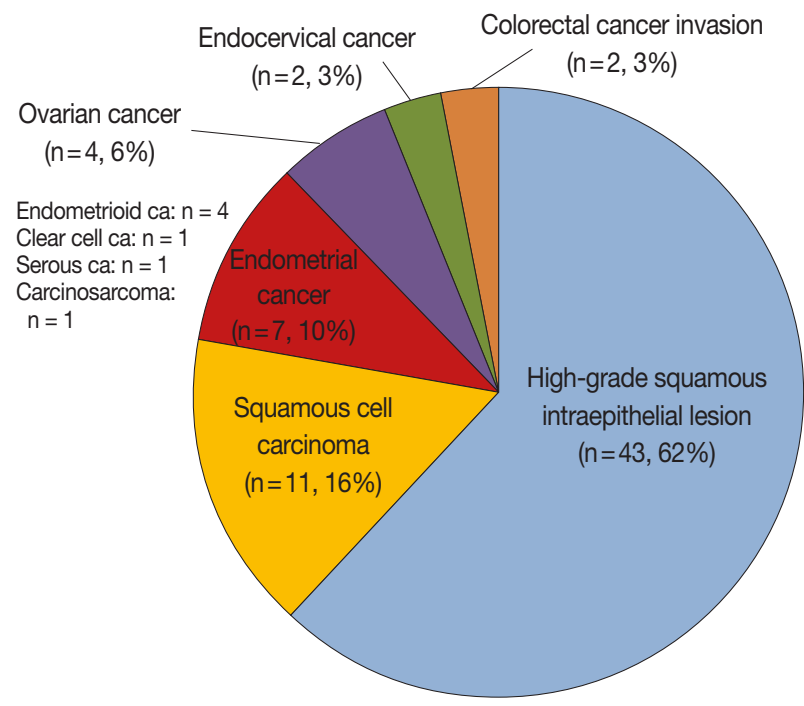

Fig. 3. Summary of the high-grade epithelial cell abnormalities cohort. The majority were squamous lesions (78\%). noma) were characterized by neoplastic cells with anisocytosis, increased nuclear:cytoplasmic ratio, and hyperchromatic nuclei with coarse chromatin (Fig. 4A, B). Tumor diathesis was seen in squamous cell carcinomas, and the presence of scattered atypical dyskeratotic cells were helpful features in recognizing the HCG as squamous (Fig. 4C). Neoplastic endocervical glandular HCGs demonstrated acinar patterns within the HCGs and the neoplastic cells were often columnar cells with pseudostratification (Fig. 4D). Smaller sheets and strips of neoplastic cells were present outside the HCGs with more obvious rosette-like patterns (Fig. 4E). The tumor cells frequently demonstrated macronucleoli, and scattered single cells were observed in one endocervical adenocarcinoma case. When the squamous and glandular HCGs were compared, squamous HCGs tended to show flattening of cells at periphery of HCG and oval-to-round nuclei (Fig. 4A, B), while the glandular HCGs demonstrated acinar patterns within the HCGs and cigar-shaped nuclei (Fig. 4D). HCGs of endometrioid carcinomas were characterized by tight clusters of smaller neoplastic cells (Fig. 4F). The nuclear pleomorphism was subtle in the well-differentiated tumors, while more obvious
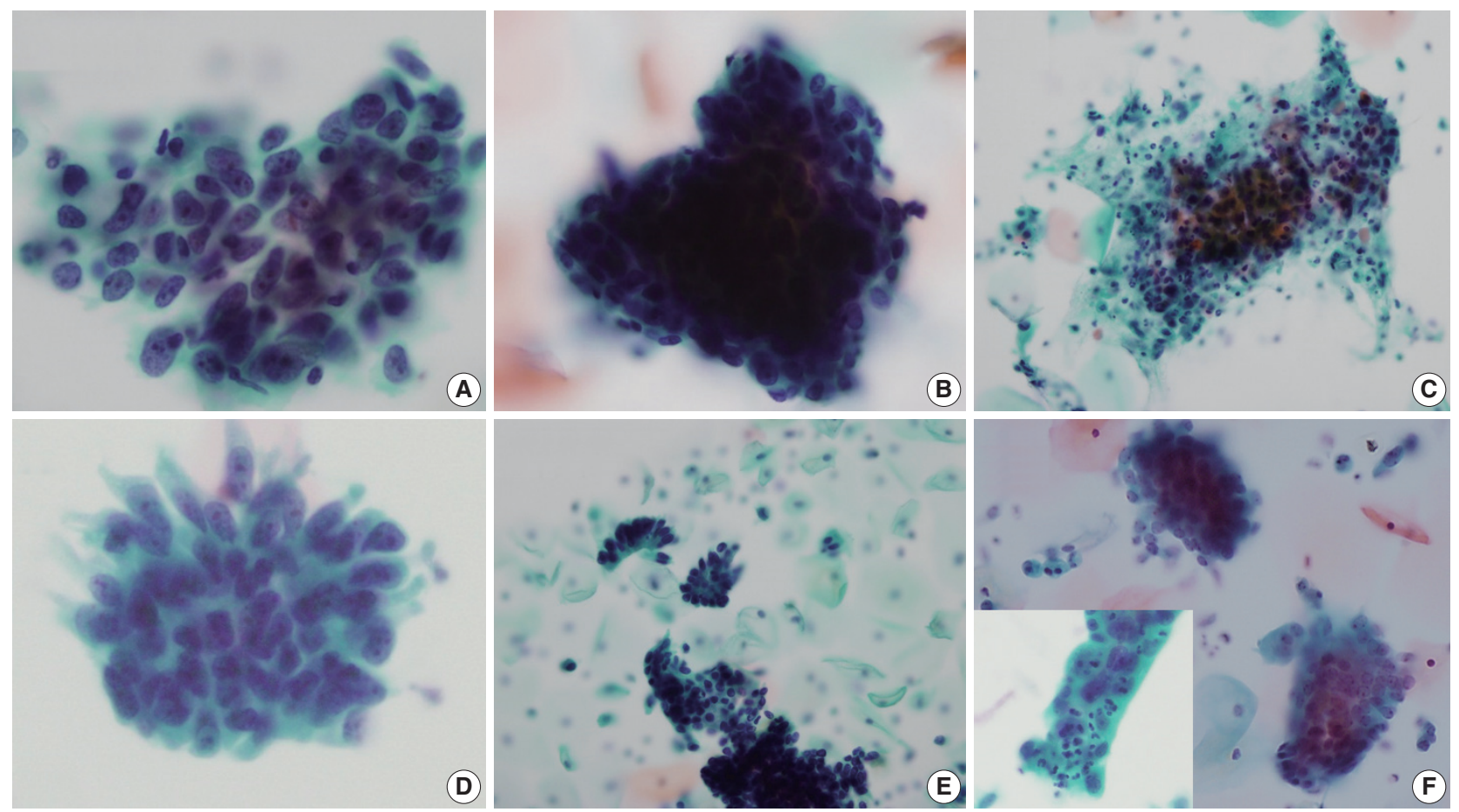

Fig. 4. Cytological features of neoplastic hyperchromatic crowed group (HCG). (A-C) HCGs of squamous cell carcinoma demonstrate dense cellular clusters with chaotic arrangement, and the individual cells are markedly hyperchromatic. The periphery of the HCG is flattened. When present, tumor diathesis and scattered dyskeratotic cells are helpful features in recognizing squamous cell carcinomas (C). (D, E) HCGs of endocervical adenocarcinomas. A vague acinar arrangement is seen within the HCG with feathering of the border (D). Smaller strips and rosettes of tumor cells are helpful clues in the diagnosis (E). (F) HCGs of well-differentiated endometrioid carcinoma were tight clusters of neoplastic cells that were smaller than endocervical adenocarcinoma. More obvious nuclear atypia were present in high-grade tumors and intracytoplasmic neutrophils were also present (inset). 
nuclear atypia was present in high-grade endometrioid carcinomas. Intracytoplasmic neutrophils ("bags of polys") were also seen in one case (Fig. 4F, inset). The ovarian and colorectal carcinoma cases all showed glandular cell clusters: the background of the ovarian cancer metastases were clean, while the two colorectal adenocarcinomas demonstrated a necrotic and inflammatory background, suggestive of direct invasion of the uterine cervix by the tumor.

When we examined the presence of individually scattered neoplastic cells in the background, invasive squamous cell carcinomas more frequently demonstrated neoplastic single cells compared to glandular neoplasia (10/11 vs $4 / 15$, respectively; $\mathrm{p}=.002$ ). Glandular lesions demonstrating scattered neoplastic cells were all primary uterine lesions ( 3 endometrial carcinomas and 1 endocervical adenocarcinoma), and extrauterine neoplasms all presented as cellular clusters on cervicovaginal cytology.

\section{DISCUSSION}

HCGs have received more attention in cervicovaginal cytology since the introduction of the LBC test, as the HCGs are more visible against the monolayer background with the LBC method, compared to conventional smears. As LBC is being increasingly used (in Korea, the proportion of cervicovaginal LBC tests has increased from $7.6 \%$ in 2004 to $25.3 \%$ in 2015), ${ }^{5,6}$ it is important to be aware that HCGs may occasionally be the causes of false-negative or false positive cytology interpretation and to become familiar with the morphological characteristics of different HCGs.

In this study, HCGs were observed in 34\% of routine cervicovaginal $\mathrm{LBC}$ samples, although less than the previously reported literature. ${ }^{2}$ The majority of HCGs were non-neoplastic cell clusters as previously reported, and the non-neoplastic HCGs were mostly parabasal cell clusters in atrophy. However, the small number of neoplastic HCGs in this study were all high-grade lesions, suggesting that HCGs in cervicovaginal LBC should be carefully evaluated in order to avoid serious false-negative interpretations. ${ }^{2-4}$ Conversely, non-neoplastic HCGs may also be overinterpreted as abnormal; for example, specimens from the lower uterine segment have been reported to be over-diagnosed as positive in patients with histories of endocervical adenocarcinomas. ${ }^{7}$ Therefore, although the cytological evaluation of HCGs is often difficult due to the dense obscuring hyperchromasia of the clusters, it is still important to discriminate between neoplastic and non-neoplastic HCGs. One important tip is to evaluate the cytomorphology at the periphery of the HCGs, where the cells may be more spread out into a single layer. For example, we observed that atrophic cells are flattened at the edges of HCGs into monolayer sheets where the streaming pattern and the bland nuclear features are more easily appreciable. As another example, endocervical adenocarcinomas may show the typical feathery pattern of columnar cells at the edge of the HCGs. Secondly, it is useful to examine each focal plane of the HCG by focusing up and down. By examining each focal plane, it is possible to appreciate the lack of nuclear overlapping in atrophic cell clusters where the parabasal-like cells are stacked in multilayer sheets, and in the case of adenocarcinoma, vague acinar patterns may be visible within the HCGs. Finally, examining the scattered cells and the background outside the HCGs is extremely important. We found scattered neoplastic cells in the majority of squamous cell carcinomas in addition to the tumor diathesis. On the other hand, scattered neoplastic cells were relatively rare in glandular lesions; however, when present, the cytological features of the scattered cells showed the typical features of glandular neoplasia which helped the diagnosis.

In this study, all cases with HCGs in the routine cohort contained EC/TZ components, while about $15 \%$ of HCG-negative cases did not demonstrate EC/TZ components. The presence of $\mathrm{EC} / \mathrm{TZ}$ components in LBC implies that the cervix has been properly sampled, and thus increasing the likelihood of detecting ECAs when present. Chivukula et al. ${ }^{2}$ reported that most HCGs were endocervical cell aggregates and that ECA was detected only when HCGs were present.

In efforts to solve the diagnostic dilemma of HCG, cell block preparations and immunohistochemistry for $\mathrm{p} 16$ have been studied on cases showing HCGs. ${ }^{8,9}$ These methods were particularly helpful in identifying neoplastic HCGs from menstrual contamination. An image analysis study of HCGs, where the area, shape and color intensity of HCGs were evaluated, demonstrated little discriminative value in defining the neoplastic or benign nature of HCGs. ${ }^{10}$

To conclude, we examined the prevalence of HCGs in routine cervicovaginal LBC and described the morphological features of different types of HCGs. Examining the edges of HCGs, each plane of focus and scattered cells outside HCGs were helpful in the interpretation of the HCGs.

\section{ORCID}

Youngeun Lee: http://orcid.org/0000-0002-0000-1615

Cheol Lee: http://orcid.org/0000-0001-5098-8529

In Ae Park: http://orcid.org/0000-0001-6667-3484 
Hyoung Jin An: http://orcid.org/0000-0002-4009-836X

Haeryoung Kim: http://orcid.org/0000-0002-4205-9081

\section{Author Contributions}

Conceptualization: HK.

Data curation: YL, HK.

Investigation: YL, HK.

Methodology: YL, HK.

Resources: YL, HK, IAP, CL, HJA.

Supervision: HK.

Visualization: YL, HK.

Writing—original draft: YL, HK.

Writing—review \& editing: YL, HK, CL, IAP.

\section{Conflicts of Interest}

The authors declare that they have no potential conflicts of interest.

\section{Funding}

This work was supported by the Basic Science Research Program through NRF funded by the Ministry of Education (NRF2016R1D1A1A09919042).

\section{REFERENCES}

1. DeMay RM. Common problems in Papanicolaou smear interpretation. Arch Pathol Lab Med 1997; 121: 229-38.

2. Chivukula M, Austin RM, Shidham VB. Evaluation and signifi- cance of hyperchromatic crowded groups (HCG) in liquid-based paps. Cytojournal 2007; 4: 2.

3. Croll E, Rana DN, Walton LJ. Hyperchromatic crowded cell groups in gynaecological liquid-based cytology samples. Br J Biomed Sci 2010; 67: 154-63.

4. Gupta N, John D, Dudding N, Crossley J, Smith JH. Factors contributing to false-negative and potential false-negative cytology reports in SurePath liquid-based cervical cytology. Cytopathology 2013; 24: 39-43.

5. Oh EJ, Jung CK, Kim DH, et al. Current cytology practices in Korea: a nationwide survey by the Korean Society for Cytopathology. J Pathol Transl Med 2017; 51: 579-87.

6. Lim SC, Yoo CW. Current status of and perspectives on cervical cancer screening in Korea. J Pathol Transl Med 2019; 53: 210-6.

7. Feratovic R, Lewin SN, Sonoda Y, et al. Cytologic findings after fertility-sparing radical trachelectomy. Cancer 2008; 114: 1-6.

8. Diaz-Rosario LA, Kabawat SE. Cell block preparation by inverted filter sedimentation is useful in the differential diagnosis of atypical glandular cells of undetermined significance in ThinPrep specimens. Cancer 2000; 90: 265-72.

9. Ge Y, Mody DR, Smith D, Anton R. p16(INK4a) and ProEx C immunostains facilitate differential diagnosis of hyperchromatic crowded groups in liquid-based Papanicolaou tests with menstrual contamination. Acta Cytol 2012; 56: 55-61.

10. Evered A, Edwards J, Powell G. Image analysis of hyperchromatic crowded cell groups in SurePath cervical cytology. Cytopathology 2013; 24: 113-22. 